\title{
QUELQUES REMARQUES SUR LES PARTICULARITÉS DU LEXIQUE FRANÇAIS AU SÉNÉGAL ${ }^{1}$
}

\author{
Jan Holeš
}

\begin{abstract}
After an introductory note on Senegal and its linguistic situation, the contribution presents different types of particularities of the French language used in Senegal, comprising, among others, words loaned from local languages, neologisms formed by derivation, composition and semantic modifications. The remaining part of the article contains some exemples of the above-mentioned particularities, accompanied with photographs and exemples excerpted directly from the Senegalese press.
\end{abstract}

Key words: Senegal; French language; Wolof; neologisms; lexicology.

Résumé : Après une brève présentation du Sénégal et sa situation linguistique, l'article présente divers types de particularités linguistiques de la langue française pratiquée au Sénégal, comprenant, entre autres, les emprunts aux langues locales, les néologismes formés par dérivation ou par composition et les modifications sémantiques. La partie suivante de l'article contient quelques exemples de ces particularités, accompagnés de photographies et d'exemples tirés directement de la presse sénégalaise.

Mots-clés : Sénégal ; langue française ; wolof ; néologismes ; lexicologie.

\section{Introduction}

La République du Sénégal est un pays francophone de l'Afrique de l'Ouest², d'une superficie de 196200 km². Il est limité par le Mali à l'est, par la Mauritanie au nord, par la Guinée-Bissau et la Guinée au sud et par l'Atlantique à l'est. Le Sénégal a également une frontière avec la Gambie, qui constitue une enclave le long des côtés du fleuve Gambie, jusqu'à $320 \mathrm{~km}$ en amont de l'embouchure.

En 2010, le pays a fêté le cinquantenaire de son indépendance. Les deux premiers présidents du Sénégal sont connus même en République tchèque : Léopold Sédar Senghor (1906-2001), écrivain et linguiste, dont deux recueils de poèmes ont été publiés en Tchécoslovaquie (Zpěry stínu, 1947, et Nokturna, 1965), et son successeur, Abdou Diouf (1935), qui, en tant que secrétaire général de l'Organisation internationale de la franco-

1 L'article a été écrit dans le cadre du projet « Le français en Afrique » avec le support de la Fondation scientifique de la République tchèque (GAČR, Francouzština v Africe, no. 405/09/0037).

2 Une région subsaharienne regroupant une quinzaine de pays côtiers au nord du golfe de Guinée et quelques pays de l'arrière-pays, parmi lesquels surtout les pays francophones (Bénin, Burkina Faso, Cameroun, Côte d’Ivoire, Guinée, Mali, Niger, Sénégal, Togo), anglophones (Gambie, Ghana, Libérie, Nigérie, Sierra Leone), hispanophones (Guinée équatoriale) et lusophones (Guinée-Bissau). 
phonie a visité, en 2010, la République tchèque (Holeš 2010). Depuis avril 2000, Abdoulaye Wade est le troisième président du Sénégal.

Il faut souligner le caractère particulièrement intéressant de la diversité linguistique de l'Afrique de l'Ouest où se mélangent les langues coloniales (le français, l'anglais, le portugais, l'espagnol) avec les centaines de langues indigènes, majoritairement de la famille des langues nigéro-congolaise (Krupa - Genzor, 1996). La région présente une concentration de langues extrêmement élevée ce qui en fait un vrai laboratoire linguistique.

La situation linguistique du Sénégal a été excellemment décrite par Dumont (1983) et, plus récemment, par Mbaya (2005). L'histoire linguistique du pays ainsi que les données démolinguistiques sont bien accessibles sur le site de Leclerc (2011). Dans le contexte tchèque, il ne faut pas omettre les travaux de Kadlec qui décrit systématiquement la politique et la situation linguistique du Sénégal et des autres pays francophones africains de la région (par ex. 2007 ou 2008).

La population sénégalaise est estimée à 11,7 millions d'habitants. Elle se compose de nombreuses ethnies. Les Wolofs sont les plus nombreux (représentant 43,7 \% de la population). Parmi les ethnies les plus importantes, mentionnons encore les Pulaar (23,2 \%) et les Sereer (14,8 \%) (Daff 1996 : 566). Les habitants parlent approximativement 35 langues indigènes, dont six ont été proclamées langues nationales et dont seulement le wolof est utilisé massivement. Selon Dumont (1983) et Mbaya (2005), cette langue est maîtrisée par 80-90\% des habitants. Le wolof, une des langues nigéro-congolaises, parlé par huit à dix millions de locuteurs, possède en Afrique de l'Ouest la fonction d'une langue véhiculaire et est en usage également en Gambie et en Mauritanie. ${ }^{3}$ Néanmoins, le français demeure la seule langue officielle du Sénégal. C'est l'unique langue visible dans la vie publique, dans les médias écrits, à la télévision et dans les documents officiels (lois, formulaires). Les langues locales sont massivement présentes dans les conversations courantes et informelles, à la radio. De plus, le wolof trouve une certaine place dans l'enseignement, surtout dans les écoles maternelles (Leclerc 2011).

En dépit de son statut officiel, le français n'est parlé que par quelques $11 \%$ de la population masculine et seulement par $1 \%$ de la population féminine (estimations des années $60 \mathrm{du} X X^{\mathrm{e}}$ siècle, des données plus récentes ne sont pas disponibles) (Dumont 1983 : Annexe 1). Il faut remarquer que cette situation est étroitement liée au taux de scolarisation qui reste relativement faible au Sénégal. Selon Dumont, les estimations officielles parlent de $43 \%$, mais les estimations officieuses font état de $28 \%$ (Dumont 1983 : 24).

\section{Le français sénégalais}

Le français pratiqué au Sénégal a fait l'objet de plusieurs études, par ex. celles de Dumont (1983 : 165-192) ou Daff (1996). Les deux auteurs soulignent l'influence du substrat national (c.-à-d. le wolof). Daff mentionne l'alternance codique entre le wolof et le français et le mélange des deux langues que l'on entend fréquemment dans la conversation quotidienne, à la radio et à la télévision. Le wolof urbain contient beaucoup de mots outils français (pour, mais, parce que) (Daff 1996 : 570-571), mais l'influence du français sur le wolof est massive dans tous les domaines du vocabulaire (Dumont 1983 : 113-163).

\footnotetext{
3 Certaines publications donnent des estimations différentes qui nous paraissent trop modestes : Ethnologue (Gordon 2005 : 182) estime la population totale wolophone à 3,56 millions.
} 
Les particularités linguistiques comprennent surtout (a) les emprunts aux langues indigènes, souvent les termes désignant des réalités africaines particulières, (b) les néologismes dérivés à partir des emprunts africains, les néologismes composés et (c) les glissements de sens. Cette énumération n'est pas exhaustive - voir la note 4 sur quelques archaïsmes du français sénégalais.

Dans le passage qui suit, nous allons choisir quelques exemples de ces particularités, en prêtant attention aux quelques expressions qui n'ont pas été répertoriées ailleurs. Celles qui ont été décrites par Dumont sont accompagnées des exemples dépouillés de la presse sénégalaise et des photographies qui permettent de se faire une meilleure idée de la réalité désignée. Les définitions sont les nôtres. Tous les exemples sont tirés du portail sénégalais http:/ /www.rewmi.com, apportant les actualités politiques, sociales et internationales. Nous n'avons retenu que les mots vifs, fréquents et incontournables lors d'un séjour au Sénégal.

\subsection{Les emprunts aux langues africaines}

Les emprunts aux langues locales représentent le groupe le plus important de sénégalismes. Cette catégorie comprend surtout les noms désignant les réalités traditionnelles, religieuses, gastronomiques, administratives, zoologiques et botaniques inconnues en France.

toubab s.m., s.f. " personne de race blanche ", mot omniprésent dans le contact des Européens avec la population sénégalaise.

L'acte de la dame Saly Dramé est vraiment grave pour ne pas dire monstrueux car voilà une mère qui n'a pas hésité à « vendre » sa fille à une toubab, sous le couvert de l'adoption, avant de se rétracter après que la famille ait opposé une fin de non recevoir à cette transaction. (http:// www.rewmi.com, 28 Novembre 2010) ${ }^{4}$

thiéboudienne (ou thiébou diène) s.m. "riz au poisson » ou yassa «plat préparé à base de poisson ou de poulet marinés dans du jus de citron, servi avec du riz (yassa au poulet, yassa au poisson)»

Chacune avec son équipe, prépare des menus africains aux immigrés qui viennent se restaurer. Du « thiébou diène » (riz au poisson) au Yassa, en passant par le couscous ou au mafé ${ }^{\text {, }}$ les pensionnaires font des va-et-vient incessants entre le restaurant, leurs chambres et le restaurant. (http:/ /www.rewmi.com, 23 Avril 2007)

\subsection{La formation néologique de mots}

Il s'agit soit des mots dérivés à partir des racines africaines (dibiterie, dibitier), cas spécifique pour le français sénégalais, soit à partir des racines françaises (essencerie). Les nouveaux composés sénégalais contiennent des unités connues en France qui peuvent être graphiquement soudées (taximan, télécentre), reliées ou non par un trait d'union (septplace, car rapide).

\footnotetext{
4 Remarquons, dans cet exemple, l'usage du mot dame qui représente un autre cas spécifique du français sénégalais. Il s'agit en fait d'un des vieux mots français réactivés au Sénégal. Dumont (1983 : 189) note que parallèlement à dame, le terme sieur est remis à l'honneur avec le sens de " Monsieur », surtout dans la langue journalistique.

5 Un plat de mouton, de poulet ou de viande de bœuf, préparé en Afrique de l’Ouest, avec de la pâte d'arachides.
} 
dibiterie s.f. « gargote où l'on vend du dibi » (le dibi « viande grillée au feu de bois », mot wolof) ou dibitier s.m. " propriétaire d'une dibiterie ».

En face et toujours sur le même lieu, une boutique, une dibiterie et un véhicule de transport en commun ont été la cible des assaillants. (http:/ / www.rewmi.com, 12 Octobre 2009)

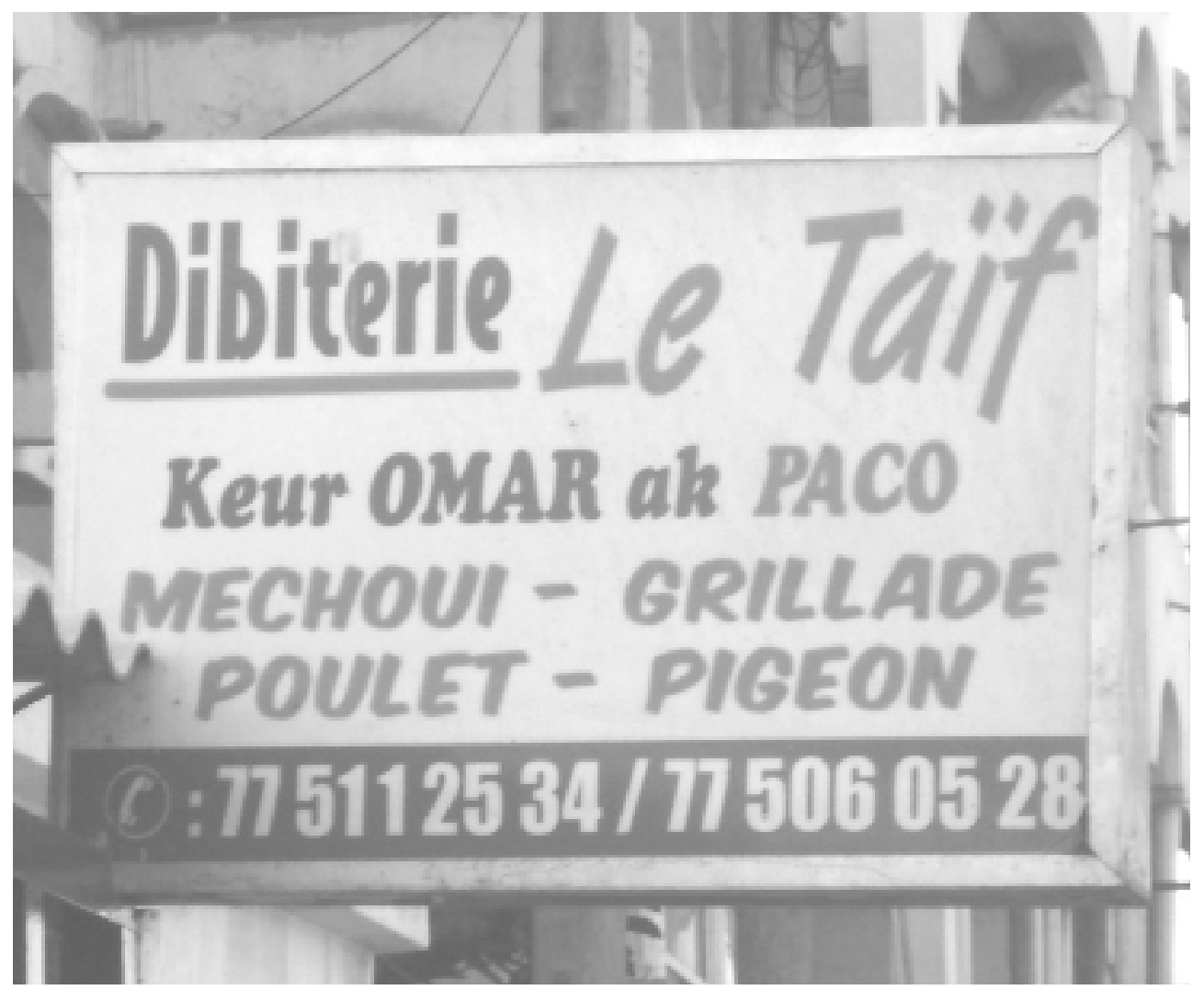

Photo 1 : Affiche d'une dibiterie dakaroise (photo de l'auteur)

essencerie s.f. « station-service»

... une voyageuse non encore identifiée qui était à bord d'un bus en provenance de Diaobé et en partance pour Dakar, a demandé la permission à un vigile de l'essencerie, d'aller dans les toilettes. Ce dernier n'y vit aucun inconvénient. Sur place cependant, la dame s'activa à autre chose. Elle accouche sans assistance, et sans le moindre scrupule, tue son bébé de sexe masculin qu'elle enveloppera dans un pagne avant de l'abandonner sur place. (http:/ / www. rewmi.com, 22 Janvier 2010)

taximan s.m. « chauffeur de taxi »

Une fois arrivé à destination, le taximan gara son véhicule devant la boutique avant de descendre pour faire sa commande, à savoir, deux sacs de riz de 50 kilos chacun et deux bidons d'huile de deux litres chacun. Une fois servi, Ousseynou ouvrit la portière de son taxi, appuya sur l'accélérateur, direction Thiès sans passer à la caisse. (http:/ / www.rewmi.com, 13 Août 2007) 
car rapide s.m. " fourgon assurant le transport en commun urbain, s'arrêtant soit à des endroits fixes, soit à la demande »

Un car rapide a heurté de manière spectaculaire la devanture d'un magasin situé sur l'avenue Blaise Diagne, blessant au passage deux passants et détériorant un lot de matériels dont un scooter. (http://www.rewmi.com, 15 Mai 2009)

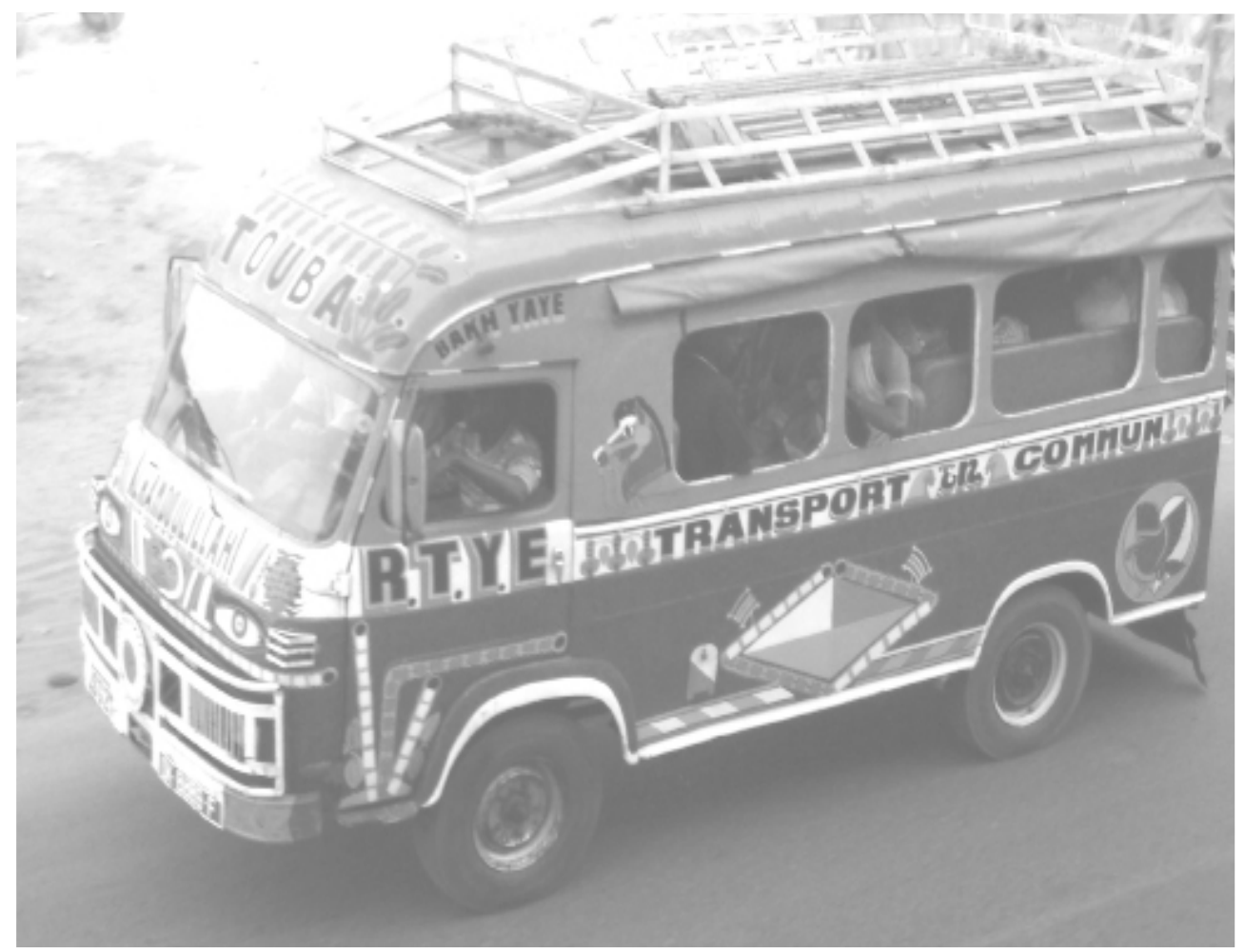

Photo 2 : Un car rapide à Dakar (photo de l'auteur)

Il est intéressant de lire le passage amusant d'un guide touristique consacré à ce moyen de transport qui est devenu un vrai symbole du Sénégal (et surtout de Dakar) :

Incroyablement décorés selon la fantaisie ou les convictions de chaque propriétaire : dessins multicolores, slogans-prières en arabe ("Faites que nous arrivions en entier »), etc. Les proclamations du style " $\mathrm{S}^{\prime}$ en fout la mort » laissent de plus en plus la place à des références religieuses, mais sont devenues leur surnom : les « s'en fout la mort » en raison du danger ambulant $[. .$.$] que représentent ces véhicules, cause de bon nombre d'accidents graves. (Le$ Sénégal + Gambie. Le guide du routard. Paris : Hachette, 2010)

sept-place s.m. "taxi collectif pour sept voyageurs, circulant sur des trajets fixes ", un autre moyen de transport sénégalais, rappelant les grands-taxis marocains ou les louages tunisiens

Collision entre 'un sept-place' et un camion sur la route de Tamba : Trois morts et plusieurs blessés graves. (http://www.rewmi.com, 19 Août 2008 ) 
télécentre s.m. "établissement privé servant pour téléphoner, envoyer des fax », aujourd'hui souvent remplacé par les cabines téléphoniques, cybercafés et les téléphones portables

En effet, les clients qui prenaient d'assaut les télécentres, pour passer un coup de fil d'urgence, ou converser avec un ami, préfèrent maintenant user du crédit à détail pour appeler à partir de leur mobile ou juste passer un message. (http:// www.rewmi.com, 25 Août 2008)

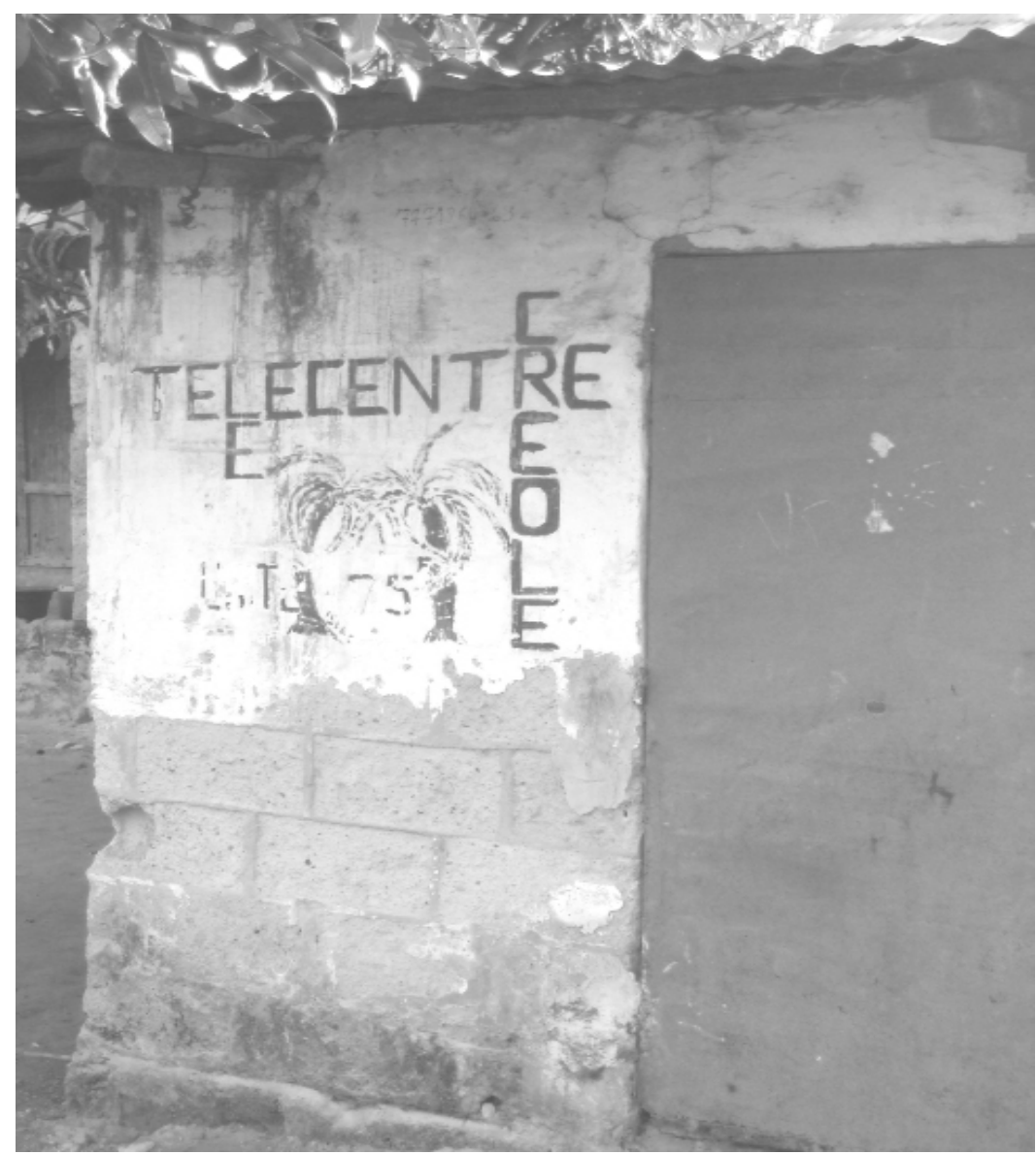

Photo 3 : Un télécentre à Ziguinchor (photo de l'auteur)

\subsection{Les modifications sémantiques}

Les changements de sens comprennent le changement de référent, les restrictions de sens, les extensions d'emploi etc.

batterie s.f. " pile ». Cette acception du mot n'est pas mentionnée par le Nouveau Petit Robert de la langue française (2011).

Le jeune homme, employé dans un magasin d'informatique, venait d'installer une nouvelle batterie dans son téléphone quand le drame s'est produit. L'un des employés du magasin 
atteste avoir entendu le bruit de l'explosion et vu son collègue étendu dans une mare de sang. La police qui a ouvert une enquête cherche à déterminer si la batterie en question n'était pas une contrefaçon. (http://www.rewmi.com, 8 Février 2009)

douche s.f. «W.C. »

"J'ai du mieux par rapport au premier jour. Je souffre à la poitrine et les mains seulement ", confie Ousmane Diao. Apparemment en forme, le jeune patient raconte son odyssée du samedi dernier. "J'habite au quartier Doumasou. Samedi dernier, j'étais à la maison, je me lavais quand une grenade m'a touché sous la douche (il s'agit de toilettes en plein air); ça m'a fait très mal. » (http://www.rewmi.com, 25 Avril 2007)

garage s.m. " gare routière de bus et de taxis collectifs »

La place de l'indépendance à Dakar transformée en garage où les taxis et clandos se disputent la clientèle. Tel est le spectacle qui s'offre aux travailleurs et aux passants en plein centre ville. (http:/ / www.rewmi.com, 30 Septembre 2010)

campement s.m. " établissement hôtelier, auberge, avec les toilettes et les douches séparées des chambres ». Dans la plupart des cas, le mot ne correspond pas au mot camping. Pour le même mot français, le Nouveau Petit Robert de la langue française (2011) donne les acceptions suivantes: (1) Action de camper. (2) Lieu, installations où l'on campe. (Un campement de Tsiganes). (3) Fig. Installation provisoire et désordonnée.

Un employé du campement a avisé son patron du comportement suspect de leurs hôtes, surtout qu'ils avaient les habits tout mouillés. Les gendarmes, mis au parfum, viendront les cueillir. (http://www.rewmi.com, 2 Juillet 2007)

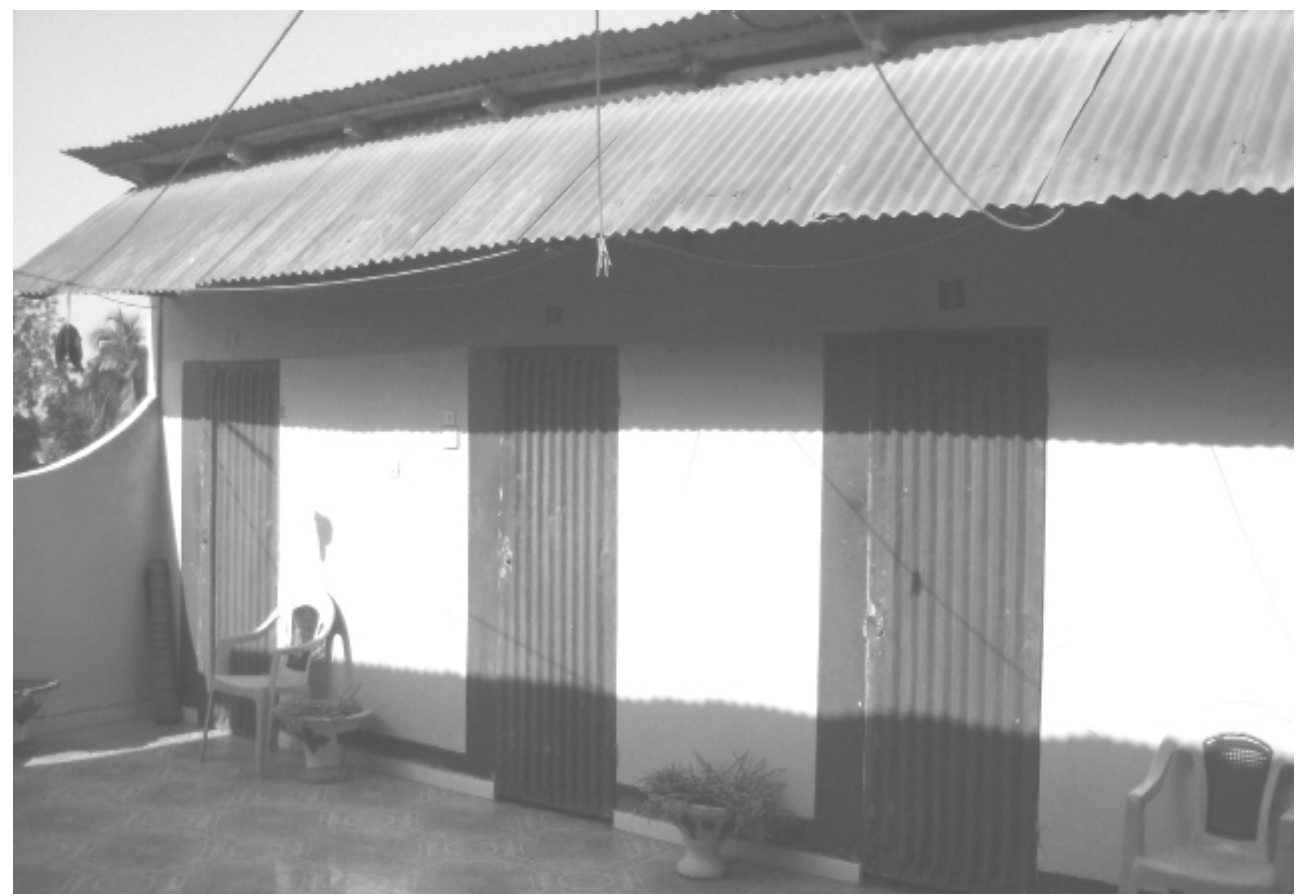

Photo 4 : Un campement à Ziguinchor 


\section{Conclusion}

Dans le français sénégalais, on trouve des particularités sur tous les niveaux de la langue. On y repère les simplifications (ou les fautes) phonétiques, morphologiques et syntaxiques, dues sans doute au fait que le français au Sénégal est une langue apprise à l'école en tant que langue étrangère. Comme dans la plupart des variantes du français un peu partout dans le monde, on y trouve des emprunts au substrat (le wolof), des néologismes formés par dérivation ou composition et des changements de sens. Nous avons énuméré quelques particularités de chaque type, en incluant des exemples originaux tirés de la presse sénégalaise et en documentant quelques-unes de ces expressions par des photographies.

\section{Bibliographie}

DAfF, Moussa (1996) : «La situation du français au Sénégal », in : Robillard, Didier de - Beniamino, Michel, éds. : Le français dans l'espace francophone. Description linguistique et sociolinguistique de la francophonie. Tome 2, Paris : Honoré Champion, p. 565-575.

Dumont, Pierre (1983) : Le français et les langues africaines au Sénégal. Paris: ACCT Karthala.

Gordon, Raymond G., éd. (2005, 15éd.) : Ethnologue. Languages of the World. Dallas : SIL International.

Holeš, Jan (2010/2011) : «Abdou Diouf à Olomouc », Romanica Olomucensia Vol. 22, No. 1, p. 91-95.

KADLEC, Jaromír (2007) : " Le français et les langues nationales au Sénégal », in : Buzek, Ivo - Veselá, Jana, éds. : Studia Romanistica 7, Ostrava : Ostravská univerzita, p. 53-58.

KADLEC, Jaromír (2008) : «Jazyková situace a postavení francouzštiny v Kamerunu », Časopis pro moderní filologii 90, p. 97-106.

KRUPA, Viktor - GENZOR, Jozef (1996) : Jazyky sveta v priestore a čase, Bratislava: Veda.

LECLERC, Jacques (2011) : L'aménagement linguistique dans le monde, http:/ / www.tlfq. ulaval.ca/AXL/, online le 4 février 2011.

Mвауа, Maweja (2005) : Pratiques et attitudes linguistiques dans l'Afrique d'aujourd'hui: le cas du Sénégal, Muenchen : LINCOM.

Nouveau Petit Robert de la langue française. Version électronique, http://lerobert. demarque.com/, en ligne le 4 février 2011.

Jan Holeš

Katedra romanistiky

Filozofická fakulta

Univerzita Palackého v Olomouci

Křížkovského 10

77180 Olomouc

République tchèque

jan.holes@upol.cz 